\title{
Morbidity and mortality associated with hypernatremia in patients with severe traumatic brain injury
}

\author{
Aditya Vedantam, MD, Claudia S. Robertson, MD, and Shankar P. Gopinath, MD \\ Department of Neurosurgery, Baylor College of Medicine, Houston, Texas
}

\begin{abstract}
OBJECTIVE Hypernatremia is independently associated with increased mortality in critically ill patients. Few studies have evaluated the impact of hypernatremia on early mortality in patients with severe traumatic brain injury (TBI) treated in a neurocritical care unit.

METHODS A retrospective review of patients with severe TBI (admission Glasgow Coma Scale score $\leq 8$ ) treated in a single neurocritical care unit between 1986 and 2012 was performed. Patients with at least 3 serum sodium values were selected for the study. Patients with diabetes insipidus and those with hypernatremia on admission were excluded. The highest serum sodium level during the hospital stay was recorded, and hypernatremia was classified as none ( $\leq$ $150 \mathrm{mEq} / \mathrm{L})$, mild (151-155 mEq/L), moderate (156-160 mEq/L), and severe (> $160 \mathrm{mEq} / \mathrm{L})$. Multivariate Cox regression analysis was performed to determine independent predictors of early mortality.
\end{abstract}

RESULTS A total of 588 patients with severe TBI were studied. The median number of serum sodium measurements for patients in this study was 17 (range 3-190). No hypernatremia was seen in 371 patients (63.1\%), mild hypernatremia in 77 patients (13.1\%), moderate hypernatremia in 50 patients $(8.5 \%)$, and severe hypernatremia in 90 patients $(15.3 \%)$. Hypernatremia was detected within the 1 st week of admission in $79.3 \%$ of patients $(n=172)$, with the majority of patients $(46 \%)$ being diagnosed within 72 hours after admission. Acute kidney injury, defined as a rise in creatinine of $\geq 0.3 \mathrm{mg} / \mathrm{dl}$, was observed in 162 patients $(27.6 \%)$ and was significantly associated with the degree of hypernatremia $(p<0.001)$. At discharge, 148 patients $(25.2 \%$ ) had died. Hypernatremia was a significant independent predictor of mortality (hazard ratios for mild: 3.4 , moderate: 4.4 , and severe: $8.4 ; p<0.001$ ). Survival analysis showed significantly lower survival rates for patients with greater degrees of hypernatremia (log-rank test, $p<0.001$ ).

CONCLUSIONS Hypernatremia after admission in patients with severe TBI was independently associated with greater risk of early mortality. In addition to severe hypernatremia, mild and moderate hypernatremia were significantly associated with increased early mortality in patients with severe TBI.

https://thejns.org/doi/abs/10.3171/2017.7.FOCUS17418

KEY WORDS severe traumatic brain injury; hypernatremia; neurocritical care; mannitol; mortality

$\mathrm{H}$ YPERNATREMIA has been shown to increase mortality in critically ill patients, independent of comorbidities. $5,13,14,17$ Although the negative impact of hypernatremia is well described for patients treated in medical or surgical ICUs, ${ }^{5,14,17}$ there is increased interest in the effects of hypernatremia in patients treated in neurocritical care units. ${ }^{2,18}$ In particular, few studies have evaluated the independent effect of hypernatremia on mortality after severe traumatic brain injury (TBI). ${ }^{6,15}$

The relationship between hypernatremia and TBI is complex, and there is considerable heterogeneity in prior studies evaluating hypernatremia in patients with TBI. ${ }^{10}$
The use of hyperosmolar therapy (mannitol and/or hypertonic saline), hypovolemia, and central diabetes insipidus are some of the factors that can produce hypernatremia in patients with TBI. Although hypertonic saline is sometimes used to treat cerebral edema, more data are needed on the consequences of inducing hypernatremia in patients with severe TBI. Presently, it is unclear if the degree of hypernatremia is associated with early outcomes in severe TBI.

The aim of the present study was to evaluate the incidence, severity, and impact of hypernatremia in a series of patients with severe TBI who were treated in a dedicated neurocritical care unit. 
TABLE 1. Baseline clinical and demographic data for 588 patients with severe TBI admitted to the neurocritical care unit

\begin{tabular}{|c|c|c|c|c|c|}
\hline Characteristic & $\begin{array}{c}\mathrm{Na} \leq 150 \mathrm{mEq} / \mathrm{L}, \\
n=371\end{array}$ & $\begin{array}{c}\text { Na } 151-155 \mathrm{mEq} / \mathrm{L} \\
\mathrm{n}=77\end{array}$ & $\begin{array}{l}\mathrm{Na} 156-160 \\
\mathrm{mEq} / \mathrm{L}, \mathrm{n}=50\end{array}$ & $\begin{array}{c}\mathrm{Na}>160 \mathrm{mEq} / \mathrm{L}, \\
\quad n=90\end{array}$ & $\begin{array}{c}p \\
\text { Value }\end{array}$ \\
\hline Age in yrs, mean \pm SD & $35.6 \pm 14.2$ & $39.4 \pm 16.5$ & $38.9 \pm 16.2$ & $34.3 \pm 13.5$ & 0.06 \\
\hline Sex & & & & & 0.94 \\
\hline Male & $310(83.6 \%)$ & $64(83.1 \%)$ & $39(78.0 \%)$ & $76(84.4 \%)$ & \\
\hline Female & $60(16.2 \%)$ & $13(16.9 \%)$ & $11(22.0 \%)$ & $14(15.6 \%)$ & \\
\hline Other & $1(0.3 \%)$ & 0 & 0 & & \\
\hline Prehospital hypoxia, $n=580$ & $44(12.0 \%), n=367$ & $13(17.1 \%), n=76$ & $17(34.7 \%), n=49$ & $17(19.3 \%), n=88$ & $<0.001$ \\
\hline Prehospital hypotension, $n=580$ & $39(10.6 \%), n=368$ & $15(19.7 \%), n=76$ & $11(22.4 \%), n=49$ & $19(21.8 \%), n=87$ & 0.006 \\
\hline Initial GCS score, mean \pm SD & $5.4 \pm 1.9$ & $5.4 \pm 2.0$ & $4.4 \pm 1.7$ & $3.8 \pm 1.4$ & $<0.001$ \\
\hline Initial motor GCS score, mean \pm SD; $n=138$ & $3.3 \pm 1.8$ & $3.0 \pm 1.8$ & $2.3 \pm 1.6$ & $1.7 \pm 1.2$ & $<0.001$ \\
\hline Bilat unreactive pupils & $91(24.5 \%)$ & $33(42.9 \%)$ & $27(54.0 \%)$ & $66(73.3 \%)$ & $<0.001$ \\
\hline ISS, mean \pm SD; $n=585$ & $29.7 \pm 9.5$ & $31.0 \pm 7.9$ & $31.0 \pm 10.1$ & $29.7 \pm 8.2$ & 0.56 \\
\hline Initial Marshall CT findings & & & & & $<0.001$ \\
\hline Diffuse injury, $n=335$ & $245(66.8 \%), n=367$ & $41(55.4 \%), n=74$ & $19(38.8 \%), n=49$ & $30(34.9 \%), n=86$ & \\
\hline Mass lesion, $n=241$ & $122(33.2 \%), n=367$ & $33(44.6 \%), n=74$ & $30(61.2 \%), n=49$ & $56(65.1 \%), n=86$ & \\
\hline No. of mannitol doses, mean \pm SD & $14.7 \pm 17.3$ & $12.6 \pm 14.3$ & $19.6 \pm 18.2$ & $17 \pm 12$ & 0.59 \\
\hline Decompressive surgery, $n=454$ & $112(30.2 \%)$ & $30(39.0 \%)$ & $21(42.0 \%)$ & $41(45.6 \%)$ & 0.02 \\
\hline Median LOS in ICU in days (IQR), $n=506$ & $13(12.8)$ & $16.8(14.2)$ & $14.9(19.9)$ & $4.9(11.2)$ & $<0.001$ \\
\hline Median LOS in hospital in days (IQR), $n=506$ & $23.0(27.0)$ & $23.5(31.0)$ & $18.0(35.0)$ & $4.0(12.0)$ & $<0.001$ \\
\hline
\end{tabular}

$I Q R=$ interquartile range; ISS = Injury Severity Score; LOS = length of stay.

Boldface type indicates statistical significance.

\section{Methods}

A research database containing data on in-patient sodium values for 700 patients with severe TBI (admission Glasgow Coma Scale [GCS] score of $\leq 8$ ) who were enrolled in prospective TBI studies from 1986 to 2012 was reviewed. Patients with at least 3 serum sodium values recorded were selected for the study. Patients with a diagnosis of diabetes insipidus or in whom desmopressin was administered were excluded from the study. Patients with hypernatremia (serum sodium $>150 \mathrm{mEq} / \mathrm{L}$ ) on admission were excluded. Treatment of patients with severe TBI was undertaken in a dedicated neurocritical ICU at Ben Taub Hospital according to the individual study protocols, but largely based on the guidelines for treatment of severe TBI at the time. Serum sodium values were obtained at least once every morning between 2 and 4 AM for all patients, and additional samples were obtained if needed throughout the day-if patients were being treated for hyper- or hyponatremia or if patients were receiving osmotic therapy. Mannitol is the only agent used for osmotic therapy in patients with intracranial hypertension at our center, and serum electrolytes were obtained more frequently in these patients during osmotic therapy.

Demographic, clinical, and imaging data obtained at admission were retrieved. The use of mannitol, the number of doses, and the timing of the last dose of mannitol were recorded. The number of sodium measurements and the timing of the highest serum sodium level were also recorded. Hypernatremia was classified based on the highest serum sodium level as follows: no hyponatremia ( $\leq$ $150 \mathrm{mEq} / \mathrm{L})$, mild (151-155 mEq/L), moderate (156-160 $\mathrm{mEq} / \mathrm{L})$, and severe (>160 mEq/L). Primary outcome was in-hospital mortality, and secondary outcome included acute kidney injury during hospital stay. Acute kidney injury was defined as a rise in the baseline creatinine by $\geq$ $0.3 \mathrm{mg} / \mathrm{dl} .{ }^{16}$

\section{Statistical Analysis}

Continuous variables were presented using descriptive statistics, with means reported as mean \pm SD. Univariate analyses for factors associated with mortality were performed using the chi-square test for categorical variables, and ANOVA for continuous variables with normal distribution. The Kruskal-Wallis test was used for continuous variables that did not fit a normal distribution. Multivariate Cox regression analysis was performed to investigate independent predictors of in-hospital mortality. Statistical software (SPSS version 20.0) was used for statistical analysis, and statistical significance was set at $\mathrm{p}<0.05$.

\section{Results}

Of the 700 patients, 14 had a diagnosis of diabetes insipidus and 72 had fewer than 3 serum sodium values measured during the hospital stay. Twenty-six patients had hypernatremia $(>150 \mathrm{mEq} / \mathrm{L})$ at admission. These patients were excluded and the remaining 588 patients were selected for analysis. Baseline demographic and clinical data for the study set are shown in Table 1. Two hundred twenty patients (37.4\%) had an initial GCS score of $3 / 15$ in the emergency room. Two hundred four patients (34.7\%) underwent decompressive surgery for TBI during the hospital stay. Two hundred thirty-two patients (39.5\%) underwent nonneurosurgical procedures and other nondecom- 
TABLE 2. Cox regression analysis to determine independent predictors of mortality after severe TBI

\begin{tabular}{lccc}
\hline \multicolumn{1}{c}{ Variable } & HR & $95 \% \mathrm{Cl}$ & $\mathrm{p} \mathrm{Value}$ \\
\hline Age & 1.01 & $1.0-1.02$ & 0.04 \\
\hline Initial motor GCS score & 0.76 & $0.66-0.87$ & $<0.001$ \\
\hline Prehospital hypoxia & 0.82 & $0.52-1.31$ & 0.41 \\
\hline $\begin{array}{l}\text { Prehospital hypotension } \\
\text { Bilat unreactive pupils (unreactive } \\
\quad \text { as compared to reactive) }\end{array}$ & 0.93 & $0.58-1.48$ & 0.76 \\
\hline $\begin{array}{l}\text { Initial Marshall CT score (mass } \\
\text { lesion as compared to diffuse) }\end{array}$ & 1.71 & $0.84-1.89$ & 0.26 \\
\hline Decompressive surgery & 0.84 & $0.55-1.28$ & 0.41 \\
\hline Hypernatremia group (mEq/L) & & & \\
\hline 151-155 & 3.4 & $1.94-5.97$ & $<0.001$ \\
\hline 156-160 & 4.4 & $2.47-8.02$ & $<0.001$ \\
\hline$>160$ & 8.4 & $5.13-13.86$ & $<0.001$ \\
\hline
\end{tabular}

Boldface type indicates statistical significance.

pressive cranial surgeries, including ventriculoperitoneal shunts. Mannitol was used in 109 patients (18.5\%), with a median of 9 doses. Comorbidities were seen in 77 patients (13.1\%). The commonest comorbidities were psychiatric illness $(\mathrm{n}=11)$, diabetes $(\mathrm{n}=9)$, HIV $(\mathrm{n}=8)$, liver disease $(\mathrm{n}=7)$, and hypertension $(\mathrm{n}=5)$.

The median number of serum sodium measurements for patients in this study was 17 (range 3-190). The highest serum sodium measurement was obtained at a median interval of 67.3 hours after the injury. Hypernatremia was detected within the 1st week of admission in $79.3 \%$ of patients $(\mathrm{n}=172)$, with the majority of patients $(46 \%)$ being diagnosed within 72 hours after admission. The highest sodium level measured during the hospital stay was $\leq 150$ $\mathrm{mEq} / \mathrm{L}$ in the majority of patients $(\mathrm{n}=371,63.1 \%)$. Acute kidney injury (rise in creatinine of $\geq 0.3 \mathrm{mg} / \mathrm{dl}$ ) during the hospital stay was noted in 162 patients $(27.6 \%)$, and this was significantly associated with the degree of hypernatremia $\left(\chi^{2}=140.2, \mathrm{p}<0.001\right)$. One hundred sixteen patients $(19.7 \%)$ had a baseline blood glucose level of $\geq 200$ $\mathrm{mg} / \mathrm{dl}$, and 264 patients $(44.9 \%)$ had a maximum blood glucose level of $\geq 200 \mathrm{mg} / \mathrm{dl}$ during the hospital stay.

Up to the time of discharge, 148 patients $(25.2 \%)$ had died. Data on the cause of death were available for 113 patients: increased intracranial pressure $(n=29)$; brain injury $(\mathrm{n}=70)$; and medical causes $(\mathrm{n}=14)$. A multivariate Cox regression model was created using age, motor GCS score at admission, presence of bilateral unreactive pupils, initial Marshall CT score, decompressive craniectomy, prehospital hypoxia, prehospital hypotension, and hypernatremia group (Table 2). Hypernatremia was significantly associated with mortality independent of age, motor GCS score, Marshall CT score, and pupil status at admission. Patients with hypernatremia $>160 \mathrm{mEq} / \mathrm{L}$ had the lowest survival rates, and patients with mild-tomoderate hypernatremia had intermediate survival rates as compared with patients without hypernatremia (Fig. 1, log-rank test; $\mathrm{p}<0.001)$. The Cox regression was repeated for patients not receiving mannitol $(\mathrm{n}=479)$, and hyper-

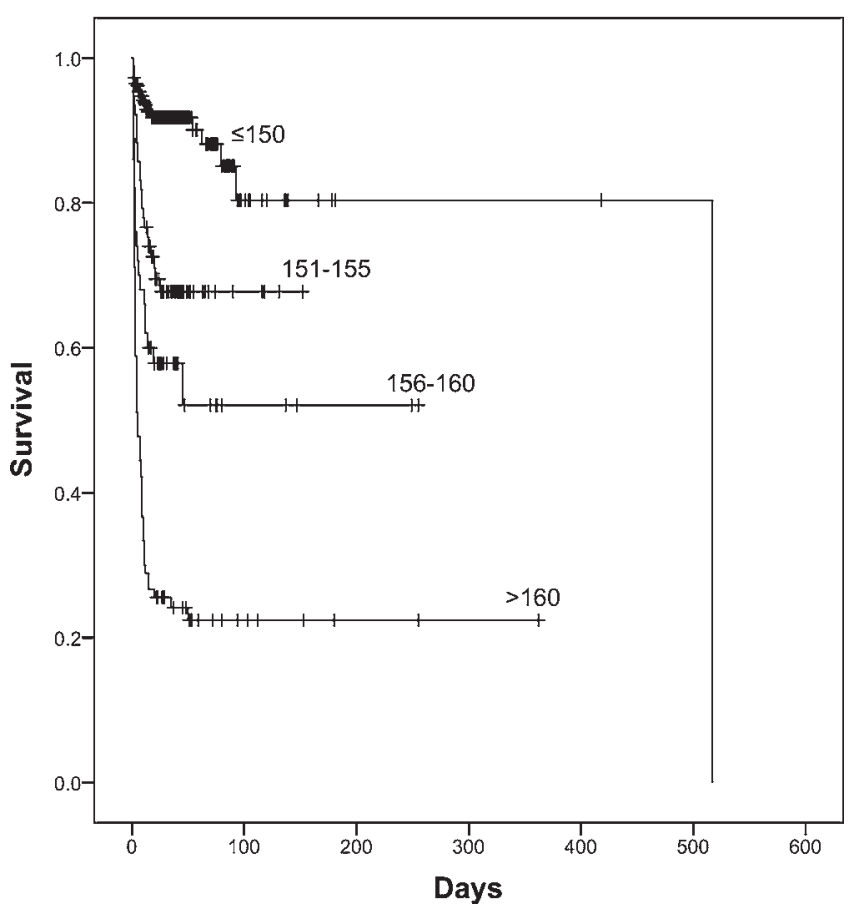

FIG. 1. Kaplan-Meier curve showing survival rates by severity of hypernatremia $(\mathrm{mEq} / \mathrm{L})$.

natremia was significantly predictive of mortality (mild: hazard ratio [HR] 3.2, p < 0.001; moderate: HR 5.1, p < 0.001 ; and severe: HR 7.9, $\mathrm{p}<0.001$ ).

\section{Discussion}

The present study evaluated the relationship between hypernatremia and early mortality after severe TBI. Hypernatremia was noted in $36.9 \%$ of patients, with the majority of the patients exhibiting mild and severe hypernatremia. Hypernatremia was independently associated with early mortality after severe TBI, with higher rates of mortality in patients with severe hypernatremia.

Hypernatremia is an important contributor to morbidity and mortality in patients with neurological disease. In patients with aneurysmal subarachnoid hemorrhage, hypernatremia has been linked to increased mortality and worse outcomes ${ }^{4,18}$ Hypernatremia in a heterogeneous group of patients treated in a neurocritical care unit was shown to be associated with higher rates of renal failure and morbidity. ${ }^{2}$ In that study, which included patients with strokes, subarachnoid hemorrhage, and TBI, severe hypernatremia $(\geq 160 \mathrm{mEq} / \mathrm{L}$ ) and not mild-to-moderate hypernatremia (151-159 mEq/L) was associated with increased mortality. However, in other studies hypernatremia, defined as sodium $\geq 150 \mathrm{mEq} / \mathrm{L}$, was associated with increased mortality in critically ill patients. ${ }^{14,17}$ Some of the heterogeneous outcomes related to hypernatremia may be due to variations in the definitions of hypernatremia as well as the patient population. Whereas some authors have defined hypernatremia as serum sodium $\geq 146 \mathrm{mEq} / \mathrm{L}, 5,18$ or $\geq 150$ $\mathrm{mEq} / \mathrm{L},{ }^{2,14,17}$ we chose to classify hypernatremia into mild, moderate, and severe, as described previously. ${ }^{2,11}$ This categorization is especially important for patients with TBI, 
because mild hypernatremia is often targeted when treating cerebral edema.

Hypernatremia demonstrates a complex relationship with TBI. ${ }^{10}$ Hypernatremia $(\geq 150 \mathrm{mEq} / \mathrm{L})$ is seen in $30 \%-$ $40 \%$ of patients with TBI. ${ }^{6,11,15,19}$ The etiology of hypernatremia may not be strictly defined in patients with TBI, and this condition may be due to hyperosmolar therapy; hypovolemia; insensible free water losses; or high sodium load in intravenous fluids, feeds, or medications. ${ }^{20}$ Additionally, hypothalamic dysfunction not resulting in overt diabetes insipidus may contribute to hypernatremia after TBI. ${ }^{15}$ In the present study, even mild and moderate hypernatremia was independently associated with increased mortality, and this relationship was maintained in patients who did not receive mannitol to treat cerebral edema. The present study raises questions about potential deleterious effects of hypernatremia. The benefits of hyperosmolar therapy in treating cerebral edema, therefore, need to be balanced against the potential morbidity associated with hypernatremia. Frequent monitoring of serum sodium levels during hyperosmolar therapy is important to avoid a rapid and substantial rise in serum sodium.

Many theories have been proposed to explain increased mortality rates in patients with hypernatremia. In the brain, hypernatremia leads to increased cellular dehydration and decreased cerebral edema, which is often the therapeutic goal in TBI. However, this altered homeostatic state can lead to myelin damage and even neuronal death. ${ }^{3}$ Hypernatremia, therefore, can contribute to additional secondary brain injury after TBI. Hypernatremia has also been shown to be associated with decreased left ventricular contractility, impaired glucose utilization, and gluconeogenesis, as well as hyperventilation and renal dysfunction. ${ }^{12}$ The association between hypernatremia and kidney injury has been shown previously. ${ }^{2,11}$ The proposed mechanism for this effect is that hypernatremia and hyperchloremia induce renal vasoconstriction that leads to a reduction in the glomerular filtration rate, and to reduced renal function. ${ }^{7,21}$ Hypotension, mannitol, and other nephrotoxic drugs can further accentuate renal dysfunction after severe TBI.

Acute hypernatremia, developing within 24 hours, can be corrected rapidly. However, hypernatremia that has persisted for a few days should be corrected at a rate of $<10 \mathrm{mEq} / \mathrm{L} / \mathrm{hr}{ }^{1}{ }^{1}$ A more rapid correction of subacute and chronic hypernatremia can lead to rebound cerebral edema and seizures. ${ }^{9}$ Calculations for the free water deficit can help determine the infusion rate of hypotonic fluids such as $0.45 \%$ sodium chloride. ${ }^{1}$ Patients who receive nasogastric feeds can be switched to a low-sodium formula in addition to scheduled free water administration. We do not have data to investigate the effects of hypernatremia correction in our series. However, rapid correction of hypernatremia was avoided due to the risk of rebound cerebral edema.

Hypernatremia is more common in patients with TBI who have central diabetes insipidus and lower admission GCS scores. ${ }^{11}$ The association between central diabetes insipidus and mortality in patients with TBI has been established previously. ${ }^{8}$ In prior studies, the link between hypernatremia and mortality may have been confounded by the presence of diabetes insipidus ${ }^{10}$ and to overcome this we excluded patients with this disease. Similar to prior studies, ${ }^{2,15}$ however, we did not have all the clinical and laboratory metrics needed to investigate milder endocrinopathy that may have contributed to hypernatremia. Additionally, patients with more severe injury (lower initial motor GCS score) were more likely to exhibit hypernatremia in the present study. Although hypernatremia was associated with the severity of injury, it remained associated with increased mortality in patients with severe TBI even when the data were adjusted for injury severity. The exact mechanism by which hypernatremia is associated with mortality in patients with TBI has yet to be explained.

The retrospective design of this study limits the evaluation of all potential outcomes related to hypernatremia. The number of patients receiving mannitol and the number of doses of mannitol received may have been underestimated due to missing medication data, and this may have affected our ability to analyze the true impact of mannitol on hypernatremia in this series. We only evaluated the highest serum sodium value and did not measure the duration of hypernatremia or the impact of hypernatremia correction. Additionally, measurements of fluid balance and osmolality might have provided additional information regarding the source of hypernatremia. The issue of residual confounding factors is important in a retrospective study like ours. The severity of injury remains a strong confounder in our study as well as in previous studies on this subject. ${ }^{10}$ Hypernatremia may have been targeted in patients with more severe cerebral edema, and these patients probably had a greater likelihood of death. Although we attempted to control for this by using a multivariate analysis, a prospective study is needed to elucidate all potential consequences of the severity and duration of hypernatremia in TBI, irrespective of injury severity. Overall, despite these limitations, this study demonstrates that hypernatremia after severe TBI may not be a benign entity, and it can potentially contribute to morbidity and mortality.

\section{Conclusions}

Hypernatremia was seen in $36.9 \%$ of patients with severe TBI who were treated in a neurocritical care unit and was independently associated with early mortality. These results highlight an additional mortality risk with hypernatremia after severe TBI.

\section{References}

1. Adrogué HJ, Madias NE: Hypernatremia. N Engl J Med 342:1493-1499, 2000

2. Aiyagari V, Deibert E, Diringer MN: Hypernatremia in the neurologic intensive care unit: how high is too high? J Crit Care 21:163-172, 2006

3. Ayus JC, Armstrong DL, Arieff AI: Effects of hypernatraemia in the central nervous system and its therapy in rats and rabbits. J Physiol 492:243-255, 1996

4. Beseoglu K, Etminan N, Steiger HJ, Hänggi D: The relation of early hypernatremia with clinical outcome in patients suffering from aneurysmal subarachnoid hemorrhage. Clin Neurol Neurosurg 123:164-168, 2014

5. Darmon M, Timsit JF, Francais A, Nguile-Makao M, Ad- 
rie $\mathrm{C}$, Cohen $\mathrm{Y}$, et al: Association between hypernatraemia acquired in the ICU and mortality: a cohort study. Nephrol Dial Transplant 25:2510-2515, 2010

6. Froelich M, Ni Q, Wess C, Ougorets I, Härtl R: Continuous hypertonic saline therapy and the occurrence of complications in neurocritically ill patients. Crit Care Med 37:14331441, 2009

7. Gerber JG, Branch RA, Nies AS, Hollifield JW, Gerkens JF: Influence of hypertonic saline on canine renal blood flow and renin release. Am J Physiol 237:F441-F446, 1979

8. Hadjizacharia P, Beale EO, Inaba K, Chan LS, Demetriades D: Acute diabetes insipidus in severe head injury: a prospective study. J Am Coll Surg 207:477-484, 2008

9. Kahn A, Brachet E, Blum D: Controlled fall in natremia and risk of seizures in hypertonic dehydration. Intensive Care Med 5:27-31, 1979

10. Kolmodin L, Sekhon MS, Henderson WR, Turgeon AF, Griesdale DE: Hypernatremia in patients with severe traumatic brain injury: a systematic review. Ann Intensive Care 3:35, 2013

11. Li M, Hu YH, Chen G: Hypernatremia severity and the risk of death after traumatic brain injury. Injury 44:1213-1218, 2013

12. Lindner G, Funk GC: Hypernatremia in critically ill patients. J Crit Care 28:216.e11-216.e20, 2013

13. Lindner G, Funk GC, Lassnigg A, Mouhieddine M, Ahmad SA, Schwarz C, et al: Intensive care-acquired hypernatremia after major cardiothoracic surgery is associated with increased mortality. Intensive Care Med 36:1718-1723, 2010

14. Lindner G, Funk GC, Schwarz C, Kneidinger N, Kaider A, Schneeweiss B, et al: Hypernatremia in the critically ill is an independent risk factor for mortality. Am J Kidney Dis 50:952-957, 2007

15. Maggiore U, Picetti E, Antonucci E, Parenti E, Regolisti G, Mergoni M, et al: The relation between the incidence of hypernatremia and mortality in patients with severe traumatic brain injury. Crit Care 13:R110, 2009

16. Molitoris BA, Levin A, Warnock DG, Joannidis M, Mehta RL, Kellum JA, et al: Improving outcomes of acute kidney injury: report of an initiative. Nat Clin Pract Nephrol 3:439-442, 2007

17. O'Donoghue SD, Dulhunty JM, Bandeshe HK, Senthuran S, Gowardman JR: Acquired hypernatraemia is an independent predictor of mortality in critically ill patients. Anaesthesia 64:514-520, 2009
18. Qureshi AI, Suri MF, Sung GY, Straw RN, Yahia AM, Saad $\mathrm{M}$, et al: Prognostic significance of hypernatremia and hyponatremia among patients with aneurysmal subarachnoid hemorrhage. Neurosurgery 50:749-756, 2002

19. Shehata M, Ragab D, Khaled M, Hegazy M, Hussein A, Khaled H: Impact of hypernatremia on patients with severe traumatic brain injury. Crit Care 14 (Suppl 1):P355, 2010

20. Tisdall M, Crocker M, Watkiss J, Smith M: Disturbances of sodium in critically ill adult neurologic patients: a clinical review. J Neurosurg Anesthesiol 18:57-63, 2006

21. Wilcox CS: Regulation of renal blood flow by plasma chloride. J Clin Invest 71:726-735, 1983

\section{Disclosures}

The authors report no conflict of interest concerning the materials or methods used in this study or the findings specified in this paper.

\section{Author Contributions}

Conception and design: Vedantam, Robertson. Acquisition of data: Vedantam, Robertson. Analysis and interpretation of data: all authors. Drafting the article: Gopinath, Vedantam. Critically revising the article: all authors. Reviewed submitted version of manuscript: all authors. Approved the final version of the manuscript on behalf of all authors: Gopinath. Statistical analysis: Vedantam. Administrative/technical/material support: Gopinath, Robertson. Study supervision: Gopinath, Robertson.

\section{Supplemental Information \\ Videos}

Video Abstract. https://vimeo.com/236605635.

\section{Previous Presentations}

Portions of this work were accepted as a poster for the 2017 National Neurotrauma Symposium.

\section{Correspondence}

Shankar P. Gopinath, Department of Neurosurgery, Baylor College of Medicine, 7200 Cambridge, Ste. 9A, MS: BCM650, Houston, TX 77030. email: shankarg@bcm.edu. 